\title{
Zur Geschichte der Gerontechnologien
}

\author{
Sabina Misoch
}

Gerontechnologie ist eine sehr junge Disziplin. Der nachfolgende Beitrag zeigt die Geschichte dieses multidisziplinären Forschungsfeldes auf und führt dabei in die wichtigsten Anwendungsbereiche von Gerontechnologien ein.

\section{Histoire des gérontechnologies}

La gérontechnologie est une discipline très récente. L'article ci-dessous présente l'histoire de ce domaine de recherche multidisciplinaire ainsi que les plus importants domaines d'application des gérontechnologies.

DOI 10.1024/2297-5160/a000044

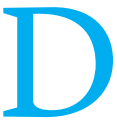

er Begriff Gerontechnology ist ein aus den englischen Wörtern gerontology und technology gebildetes Kunstwort und wurde zuerst von Jan Graafmans der Eindhoven University of Technology in die Diskussion gebracht (Graafmans et al., 1998). Das Forschungsfeld der Gerontechnologien untersucht das $\mathrm{Zu}$ sammenwirken zweier gesellschaftlicher Metatendenzen: dem demografischen Wandel (Zunahme der Lebenserwartung und rückgängige Geburtenraten) und der Dynamik der Technikentwicklung.

\section{Alter und Technik: Historisch betrachtet}

Das Forschungsfeld entstand in den 1980er Jahren, hatte zu Beginn visionären Charakter und entwickelte Ideen zur Rolle von Technik im Alltag älterer Menschen. In den 1990er Jahren etablierten sich Gerontechnologien sowie die gerontechnologische Forschung, verschiedene gerontechnische Prototypen wurden empirisch getestet und es fanden erste internationale Kongresse statt. Technische Fortschritte führten ab Mitte der 1990er Jahre zu intensiveren Forschungsaktivitäten und zu einer Ausweitung der Themengebiete. Seit 2000 bildet die Gerontechnologie ein gereiftes Forschungsfeld mit eigenen Lehrbüchern, das zahlreiche Teildisziplinen umfasst und nun stärker strukturiert und intern ausdifferenziert ist (Pelizäus-Hoffmeister, 2013).

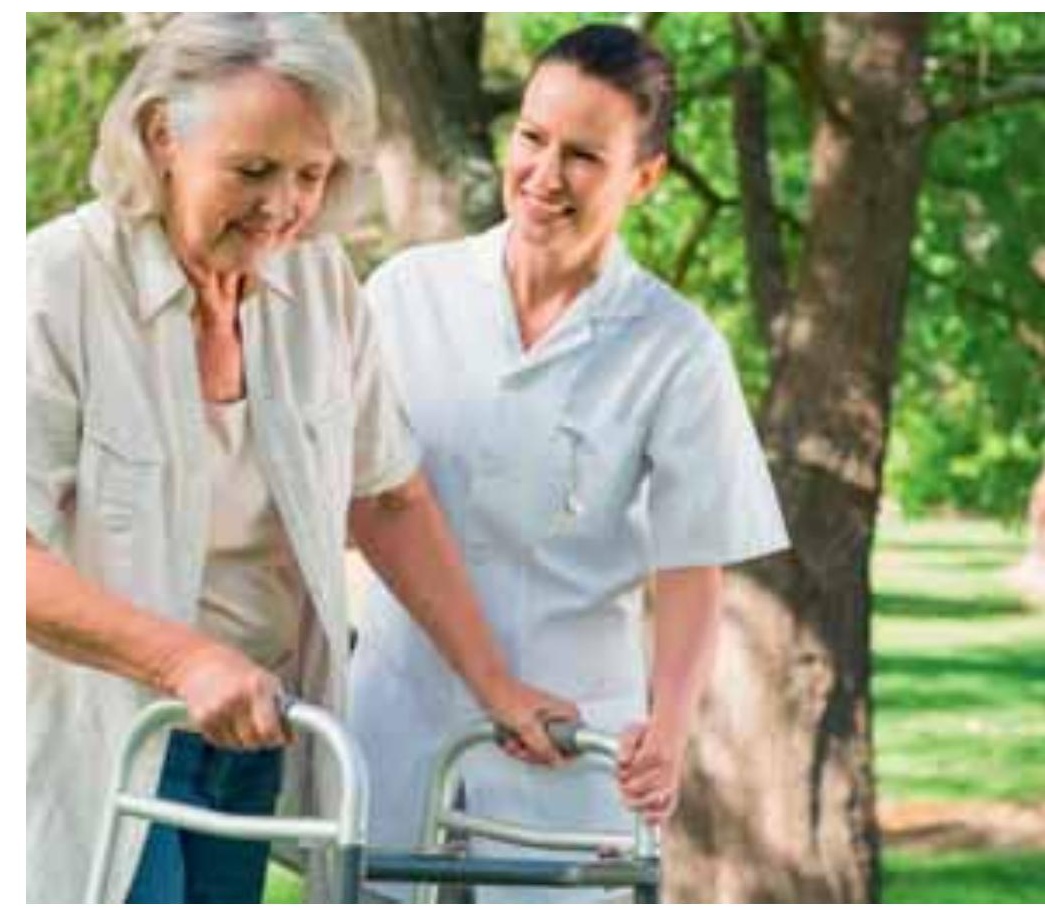

\section{Moderne Gerontechnologie}

Heute umfasst der Begriff der Gerontechnologien alle Technologien und technischen Assistenzsysteme, die ein aktives, unabhängiges und selbstständiges Altern unterstützen, indem diese altersbedingte kognitive, sensorische oder physische Einschränkungen kompensieren und/oder die individuellen Potenziale fördern und erhalten. Die Gerontechnologie befasst sich auch mit der nutzerfreundlichen und altersgerechten Gestaltung von Technologien. Ziel der Gerontechnologie ist es, die Wissensvorräte verschiedener Disziplinen «in einem konzeptuellen Rahmen $\mathrm{zu}$ integrieren und damit eine systematische Forschung» (Pelizäus-Hoffmeister, 2013, S. 92) für die Entwicklung altersgerechter Technologien voranzutreiben.

Im Zuge des demografischen Wandels und der abnehmenden personellen Ressourcen für Betreuungsleistungen werden zunehmend auch komplexe Gerontechnologien wie z.B. Anwendungen des Ambient Assisted Living (AAL) diskutiert, da mit diesen ein längeres autonomes und selbstständiges Leben im eigenen Zuhause ermöglicht wird (Misoch, 2015). Als Anwendungen der Gerontechnologien können folgende Bereiche differenziert werden: 


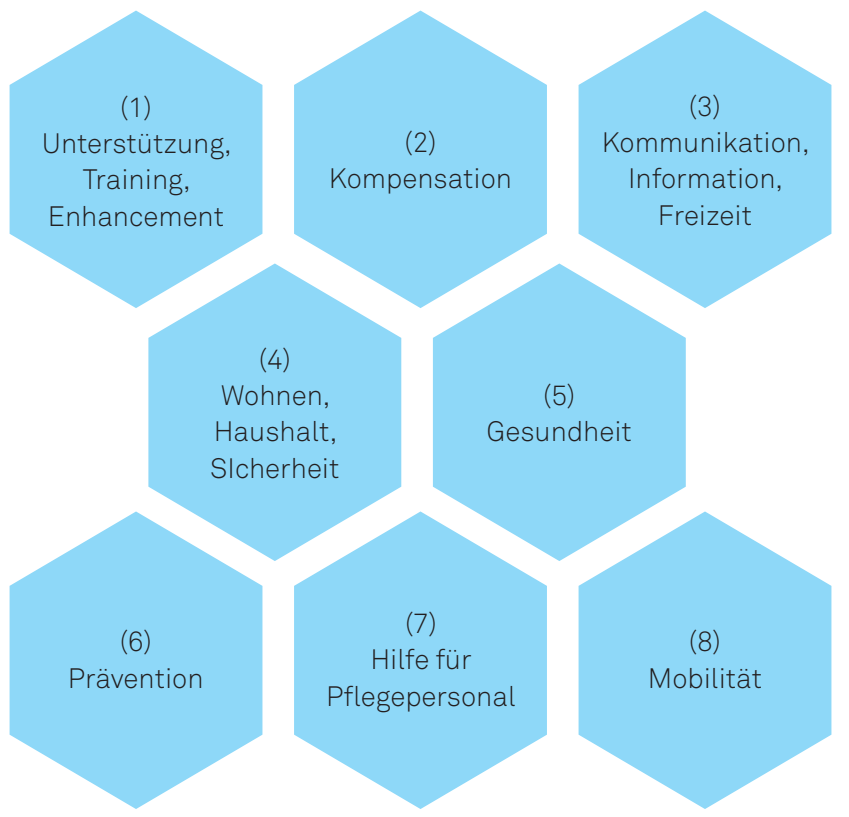

Schaubild 1. Anwendungsbereiche von Gerontechnologien

\section{Forschungsbereiche}

Die Forschungsfelder der Gerontechnologie sind dabei zu differenzieren in (a) Transporttechniken zur Erhaltung von Mobilität ausserhalb der Wohnung (in den Anwendungsbereichen 1, 2, 5 und 8), (b) Techniken, die der Gesundheitsförderung dienen (Bereiche 1, 5, 6 und 8), (c) Kommunikationstechnologien zur Pflege sozialer Kontakte, zur Informationsrecherche, zur Unterhaltung oder zum Training kognitiver Fertigkeiten (Bereiche 1, 2, 3 und 5), (d) Haushalts- und Gebäudetechniken (Bereiche 2, 4, 5, 7 und 8), (e) assistive Systeme oder Technologien, welche die Kompensation sensorischer, kognitiver oder physischer Einbussen erlauben (Bereiche 2, 4, 5, 7 und 8) sowie der Bereich der (f) Robotertechnologie (Bereiche 2, 4 und 7).

Hierbei werden Technologien auf drei Komplexitätsebenen unterschieden (nach Regnier \& Pynoos, 1992):
1. Low technology: Diese Ebene umfasst alle einfachen Gerontechnologien wie Haltegriffe oder Haltestangen.

2. Mid-level technology: Hierunter werden Produkte verstanden, die bereits existieren und die an Anforderungen älterer Menschen angepasst werden (Küchen, Rollstühle etc.).

3. High level technology: Robotertechniken, AAL sowie alle Anwendungen, die komplex und vernetzt sind (z.B. intelligente Notrufsysteme).

Gerontechnologie ist ein multidisziplinäres Feld, in dem nicht nur verschiedene wissenschaftliche Disziplinen wie Gerontologie, Architektur, Neurowissenschaften, Soziologie, Psychologie oder Medizin zusammenwirken, sondern in welches auch Designer, Technikentwickler und Industriepartner einbezogen werden müssen. Die Forschung wird heute zunehmend im Rahmen internationaler Forschungsprojekte geprägt.

\section{Literatur}

Graafmans, J.A., Taipale, V. \& Charness, N. (1998) Gerontechnology: A sustainable investment in the future (Vol. 48). Amsterdam: IOS press.

Misoch, S. (i.Dr.) AAL: Ambient Assisted Living. Chancen für ein gesundes Altern zu Hause. Therapeutische Umschau, 72 (9).

Peine, A. (2006) Innovation und Paradigma. Epistemische Stile in Innovationsprozessen. Bielefeld: Transcript.

Pelizäus-Hoffmeister, H. (2013) Zur Bedeutung von Technik im Alltag Älterer: Theorie und Empirie aus soziologischer Perspektive (Vol. 24). Wiesbaden: Springer-Verlag.

Regnier, V. \& Pynoos, J. (1992) Environmental interventions for cognitively impaired older persons. In: J.E. Birren et al. (eds.), Handbook of mental health and aging, (S. 763-792). San Diego: Academic Press.

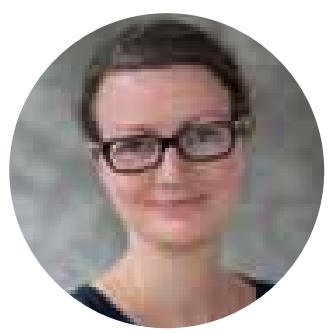

Prof. Dr. Sabina Misoch ist Leiterin des Interdisziplinären Kompetenzzentrums Alter (IKOA) an der FHS St. Gallen.

sabina.misoch@fhsg.ch 